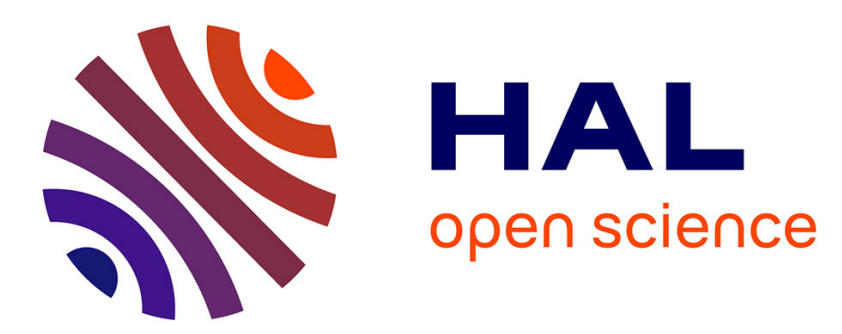

\title{
Grands Graphes De Degré Et Diamètre Fixés
}

Jean-Claude Bermond, Charles Delorme, Jean-Jacques Quisquater

\section{To cite this version:}

Jean-Claude Bermond, Charles Delorme, Jean-Jacques Quisquater. Grands Graphes De Degré Et Diamètre Fixés. Annals of Discrete Mathematics, 1983, 17, pp.65-73. 10.1016/S0304-0208(08)733747. hal-02435537

\section{HAL Id: hal-02435537 https://hal.inria.fr/hal-02435537}

Submitted on 13 Jan 2020

HAL is a multi-disciplinary open access archive for the deposit and dissemination of scientific research documents, whether they are published or not. The documents may come from teaching and research institutions in France or abroad, or from public or private research centers.
L'archive ouverte pluridisciplinaire HAL, est destinée au dépôt et à la diffusion de documents scientifiques de niveau recherche, publiés ou non, émanant des établissements d'enseignement et de recherche français ou étrangers, des laboratoires publics ou privés. 


\title{
GRANDS GRAPHES DE DEGRÉ ET DIAMÈTRE FIXÉS
}

\author{
J.-C. BERMOND and C. DELORME
}

Université Paris-Sud, Informatique, Bât. 490, ERA 452 du C.N.R.S., 91405 Orsay, France

\section{J.-J. QUISQUATER}

Philips Research Laboratory, Av. van Becelaere 2, B-1170 Brussels, Belgium

The following problem arises in telecommunication networks: given $\Delta$, the maximum degree, and $D$, the diameter, find graphs having the greatest possible number of vertices. We give here two constructions improving earlier results and we present an extended table with the best known values.

\section{Introduction}

Soit $G=(X, E)$ un graphe simple, non orienté, où $X$ désigne l'ensemble des sommets et $E$ celui des arêtes. La distance entre deux sommets $x$ et $y$ de $G$, notée $\delta(x, y)$, est la longueur d'une plus courte chaîne entre $x$ et $y$. Le diamètre $D$ du graphe est défini par $D=\max _{(x, y) \in x^{2}} \delta(x, y)$. Le degré $d(x)$ du sommet $x$ est le nombre de sommets adjacents à $x$ et $\Delta$ désigne le degré maximum de $G$.

Nous nous intéressons au problème suivant (appelé ' $(\Delta, D)$-graph problem' dans la littérature): trouver le nombre maximum $n(\Delta, D)$ de sommets d'un graphe de degré maximum $\Delta$ et de diamètre $D$. Ce problème se pose dans les réseaux de communications (ou réseaux de microprocesseurs): les sommets représentent les stations ou processeurs et les arêtes, les liaisons bilatérales entre ces stations; le degré d'un sommet est le nombre de canaux bidirectionnels d'une station et le diamètre représente le maximum de liaisons (ou de stations intermédiaires plus un) à utiliser pour transmettre un message.

Ce problème semble avoir été considéré pour la première fois dans la littérature par Elspas [11]. La table de Storwick [19] résume les diverses contributions jusqu'en 1970. Récemment, ces résultats ont été améliorés par divers auteurs, e.g. Memmi et Raillard [16], Arden et Lee [1], Leland, Finkel, Qiao, Solomon et Uhr [15] et les auteurs de [4], [5], [9], [10], [18].

Une borne théorique sur $n(\Delta, D)$ a été donnée par Moore (voir [6] ou [7])

$$
n(2, D) \leqslant 2 D+1
$$

et pour $\Delta>2$, 


$$
n(\Delta, D) \leqslant\left(\Delta(\Delta-1)^{D}-2\right) /(\Delta-2) .
$$

Il existe des graphes, appelés graphes de Moore, pour lesquels il y a égalité dans la formule ci-dessus: il s'agit des $(2 D+1)$-cycles pour $\Delta=2$, des $(\Delta+1)$-cliques pour $D=1$, du graphe de Petersen à 10 sommets pour $\Delta=3$ et $D=2$, du graphe d'Hoffman-Singleton à 50 sommets pour $\Delta=7$ et $D=2$ et, peut-être, d'un graphe à 3250 sommets pour $\Delta=57$ et $D=2$.

La recherche de la valeur exact de $n(\Delta, D)$ apparaît comme un problème difficile (voir [3]). On s'esî donc intéressé à construire des graphes de degré maximum et diamètre donnés ayant un grand nombre de sommets. Le but de cet article est de donner la table des meilleures valeurs connues actuellement. Une bonne partie provient d'autres articles, essentiellement [4], [5], [9], [10] et [18]. De plus, nous donnons deux théorèmes nouveaux, qui permẹttent d'améliorer certaines valeurs.

\section{Nouvelles constructions}

Théorème 1. Soit $G_{1}$, un graphe dont l'ensemble $X$ des sommets a n, éléments, de diamètre $D_{1}$ et de degré maximum $\Delta_{1}$. Soit $\Delta \geqslant \Delta_{1}$. Soit $G_{2}$ un graphe à $n_{2}$ sommets, de diamètre $D_{2}$ et de degré maximum au plus $n_{1} \Delta-\sum_{x \in X} d_{1}(x)$, où $d_{1}$ est le degré dans $G_{1}$. Alors, il existe un graphe, noté $G_{1}\left[G_{2}\right]$, ayant $n_{1} n_{2}$ sommets, de degré maximum $\Delta$ et de diamètre au plus $\left(D_{1}+1\right)\left(D_{2}+1\right)-1$.

Pirenve. Soit $X$ (resp. $Y$ ) l'ensemble des sommets de $G_{1}$ (resp. $G_{2}$ ). Le graphe $G$ aura pour ensemble de sommets le produit $X \times \mathbb{Y}$. Les arêtes sont de deux sortes:

(1) Les arêtes $\left\{(x, y)\left(x^{\prime}, y\right)\right\}$ avec $\left\{x, x^{\prime}\right\}$ arête de $G_{1}$ (on a donc $n_{2}$ copies de $\left.G_{1}\right)$.

(2) Pour décrire l'autre sorte d'arêtes, associons à chaque sommet y de $\mathbb{G}_{2}$ une application $f_{y}: \Gamma_{2}(y) \rightarrow X$ telle que pour tout $x$ on ait $\left|f_{y}^{-1}(x)\right| \leqslant \Delta-d_{1}(x)$. Une telle application existe car $\left|\Gamma_{2}(y)\right| \leqslant \Delta_{2} \leqslant \sum_{x \in x}\left(\Delta-d_{1}(x)\right)$. Les arêtes de la deuxième sorte sont alors du type $\left\{\left(f_{y}\left(y^{\prime}\right), y\right),\left(f_{y} \cdot(y), y^{\prime}\right)\right\}$, où $\left\{y, y^{\prime}\right\}$ est une arête de $G_{2}$.

Le graphe $G$ ainsi obtenu a $n_{1} n_{2}$ sommets; son degré maximum est au plus $\Delta$ (le degré du sommet $(x, y)$ est $\left.d_{1}(x)+\left|f_{y}^{-1}(x)\right| \leqslant \Delta\right)$. Cherchons son diamètre. Soient $(x, y)$ et $\left(x^{\prime}, y^{\prime}\right)$ deux sommets du graphe. Il existe une chaine de longueur $\mathbb{D} \leqslant \mathbb{D}_{2}^{\prime}$ reliant $y$ à $y^{\prime}$ dans $G_{2}$; soit $y=y_{0}, y_{1}, \ldots, y_{D}=y^{\prime}$ cette chaîne. $\mathrm{La}$ distance de $\left(f_{y_{1}}\left(y_{i-1}\right), y_{i}\right)$ à $\left(f_{y_{i}}\left(y_{i+1}\right), y_{i}\right)$ est au plus $D_{1}$, de même que la distance de $(x, y)$ à $\left(f_{y}\left(y_{1}\right), y\right)$ et celle de $\left(x^{\prime}, y^{\prime}\right)$ à $\left(f_{y^{\prime}}\left(y_{D-1}\right), y^{\prime}\right)$. On a ainsi formé un chemin de longueur au plus $D_{1}(D+1)+D$ entre $(x, y)$ et $\left(x^{\prime}, y^{\prime}\right)$; le diamètre est, donc, au plus $D_{1}\left(D_{2}+1\right)+D_{2}=\left(D_{1}+1\right)\left(D_{2}+1\right)-1$. 
Exemples. (Les notations utilisées sont détaillées à la fin de cet article.)

En Fig. 1, nous avons représenté le graphe $G=G_{1}\left[G_{2}\right]$, avec

$$
G_{1}=\left[\begin{array}{lll}
0 & \\
1 & 0_{3}
\end{array}\right]
$$

et $\mathbb{G}_{2}=\mathbb{K}_{7}, \Delta=3$. Les sommets de $K_{7}$ sont notés $0,1, \ldots, 6$ et $f_{y}\left(y^{\prime}\right)=\lceil z / 2\rceil$ où $1 \leqslant z \leqslant 6$ et $y+z \equiv y^{\prime}(\bmod 7)$. Le graphe $G$, de diamètre 5 , a 28 sommets de degré 3 .

Dans le cas particulier où $G_{2}$ est le graphe complet, on retrouve une construction due à Quisquater [18]: par exemple, en prenant pour $G_{1}$ le graphe d'Hoffman-Singleton à 50 sommets, de degré 7 et de diamètre 2 , et pour $G_{2}$, le graphe $K_{51}$, on obtient un graphe à 2550 sommets noté $H S\left[K_{51}\right]$, de degré maximum 8 et de diamètre 5 . Ce dernier graphe a aussi été trouvé par Uhr (voir [15]).

D'autres résultats sont donnés à la Table 3: par exemple, soient $G_{1}$ le graphe d'Hoffman-Singleton et $G_{2}$ le graphe $P_{149}^{\prime}$ obtenu comme graphe quotient du graphe d'incidence du plan projectif $P$ d'ordre 149 ; le graphe $G_{2}$ a $149^{2}+149$ $+1=22351$ sommets, de degré $\Delta_{2}=150$, et est de diamètre 2 . Soit $\Delta=10$, $n_{1}(\Delta-7)=150=\Delta_{2}$. Le graphe $G$ est de degré 10 , de diamètre 8 et a 1117550 sommets: cette valeur est à comparer avec le graphe à 19305 sommets de la table de Storwick [19] ou avec le graphe à 745104 sommets de Bermond, Delorme et Farhi [4].

Si on prend pour $G_{1}$ le graphe à 4680 sommets de notre table 3 , de degré 12 et diamètre 4 , on peut montrer que ce graphe a 27820 arêtes. Soit $\Delta=13$ et $G_{2}=\mathbb{K}_{5201}$. On a $\Delta_{2}=5200$ et $n_{1} \Delta-\sum d(x)=60840-55640=5200$. Le graphe $G$ a donc 24340680 sommets et est de diamètre 9 .

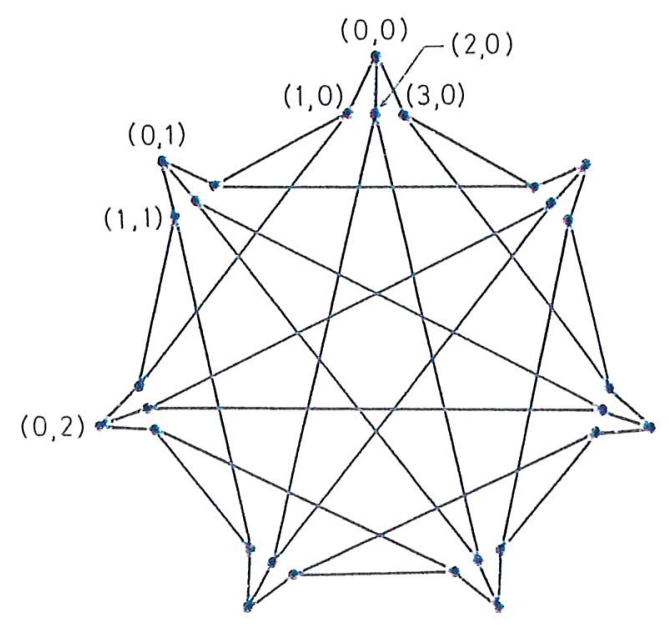

Fig. 1. 
D'autres exemples figurent encore dans la Table 3. Pour cela, nous avons besoin de connaître les valeurs de $t=n_{1} \Delta_{1}-\sum_{x \in X} d_{1}(x)$ pour certains graphes: par example,

$$
t\left(P_{q}^{\prime}\right)=q+1, \quad t\left(Q_{q}^{\prime}\right)=q^{2}+1,
$$

pour $G_{1}$, le graphe à 715 sommets, de degré 11 et de diamètre 3 , on a $t\left(G_{1}\right)=585$,

pour $G_{1}$, le graphe à 910 sommets, de degré 14 et de diamètre 3 , on a $t\left(G_{1}\right)=2340$.

Remarque. Le choix de la fonction $f$ permet bien souvent de construire plusieurs graphes différents ayant les mêmes paramètres.

Corollaire. Lorsque le degré $\Delta \rightarrow \infty$, alors $\liminf \left(n(\Delta, 5) \Delta^{-5}\right) \geqslant 4^{4} / 5^{5}$.

Preuve. Prenons pour graphe $G_{1}$, le graphe $P_{q}^{\prime}$ associé à un plan projectif de diamètre 2 , de degré $q+1$ et ayant $q^{2}+q+1$ sommets: un tel graphe existe si $q$ est une puissance de premier. Posons $q+1=4 \Delta / 5$. Pour $G_{2}$, prenons le graphe complet sur $\Delta\left(q^{2}+q+1\right) / 5+1$ sommets. On a bien $\Delta_{2} \leqslant$ $\left(q^{2}+q+1\right) \Delta-4 \Delta\left(q^{2}+q+1\right) / 5$. Le graphe $G_{1}\left[G_{2}\right]$ est de degré maximum $\Delta$, de diamètre 5 et a $\Delta\left(q^{2}+q+1\right)^{2} / 5+q^{2}+q+1=\left(4^{4} / 5^{5}\right) \Delta^{5}+\varepsilon(\Delta) \Delta^{5}$ sommets où $\varepsilon(\Delta) \rightarrow 0$ quand $\Delta \rightarrow \infty$. Donc, $\liminf \left(n(\Delta, 5) \Delta^{-5}\right) \geqslant 4^{4} / 5^{5}$.

Notons que la meilleure borne connue auparavant était 1/32 [10].

Théorème 2. Soient $G_{1}$ et $G_{1}^{\prime}$ deux graphes ayant pour ensembles de sommets respectivement $X$ et $X^{\prime}$, avec $|X|=n_{1}$ et $\left|X^{\prime}\right|=n_{1}^{\prime}$, de degrés maxima $\Delta_{1}$ et $\Delta_{1}^{\prime}$ et de diamètres $D_{1}$ et $D_{1}^{\prime}$, respectivement. Soient $\Delta>\Delta_{1}$ et $\Delta>\Delta_{1}^{\prime}$. Soit $G_{2}$ un graphe biparti, ayant pour ensemble de sommets $Y \cup Y^{\prime}$ avec $Y, Y^{\prime}$ stables et $|Y|=n_{2}$ et $\left|Y^{\prime}\right|=n_{2}^{\prime}$, de diamètre $D_{2}$ et tel que le degré d'un sommet de $Y$ soit compris entre $n_{1}$ et $n_{1} \Delta-\sum_{x \in X} d_{1}(x)$ et le degré d'un sommet de $Y^{\prime}$ soit compris entre $n_{1}^{\prime}$ et $n_{1}^{\prime} \Delta-\sum_{x^{\prime} \in X^{\prime}} d_{1}^{\prime}\left(x^{\prime}\right)$ (où $d_{1}$ (resp. $d_{1}^{\prime}$ ) désigne le degré dans $G_{1}$ (resp. $\left.G_{1}^{\prime}\right)$ ). Alors on peut construire un graphe noté $\left(G_{1}, G_{1}^{\prime}\right)\left[G_{2}\right]$ ayant $n_{1} n_{2}+n_{1}^{\prime} n_{2}^{\prime}$ sommets, de degré maximum $\Delta$ et de diamètre au plus $D_{2}+\left\lceil D_{2} / 2\right\rceil \sup \left(D_{1}, D_{1}^{\prime}\right)$ $+\left\lceil D_{2} / 2\right\rceil \inf \left(D_{1}, D_{1}^{\prime}\right)$.

Prenve. L'ensemble des sommets est constitué par la réunion disjointe de $X \times Y$ et $X^{\prime} \times Y^{\prime}$. Les arêtes sont de deux sortes:

Les arêtes $\{(u, y),(v, y)\}$ où $y$ est un sommet de $G_{2}$ et $\{u, v\}$ une arête de $G_{1}$ ou $G_{1}^{\prime}$. On a donc $n_{2}$ copies de $G_{1}$ et $n_{2}^{\prime}$ copies de $G_{1}^{\prime}$. 
A chaque sommet $y$ de $Y$, associons une application $f_{y}: \Gamma_{2}(y) \rightarrow X$ telle que pour tout $x$ de $X, 1 \leqslant\left|f_{y}^{-1}(x)\right| \leqslant \Delta-d_{1}(x)$. De même, à chaque sommet $y^{\prime}$ de $Y^{\prime}$, associons une application $f_{y}^{\prime}: \Gamma_{2}^{\prime}\left(y^{\prime}\right) \rightarrow x^{\prime}$ telle que pour tout $x^{\prime}$ de $X^{\prime}$, $1 \leqslant\left|f_{y^{\prime}}^{-1}\right| \leqslant \Delta-d_{1}^{\prime}\left(x^{\prime}\right)$. De telles applications existent d'après les conditions sur les degrés de $G_{2}$. Les arêtes de la deuxième sorte sont les arêtes $\left\{\left(f_{y}\left(y^{\prime}\right), y\right),\left(f_{y^{\prime}}(y), y^{\prime}\right)\right\}$ où $\left\{y, y^{\prime}\right\}$ est une arête de $G_{2}$.

Le graphe ainsi formé a $n_{1} n_{2}+n_{1}^{\prime} n_{2}^{\prime}$ sommets, de degré maximum $\Delta$ car le degré d'un sommet $(x, y)$ est $d(x)+\left|f_{y}^{-1}(x)\right|$. Cherchons son diamètre. Soient $(x, y)$ et $\left(x^{\prime}, y^{\prime}\right)$ deux sommets du graphe. S'il existe dans $G_{2}$ une chaîne entre $y$ et $y^{\prime}$ de longueur $D \leqslant D_{2}-1$, on pourra trouver une chaîne de longueur au plus $\lceil(D+1) / 2\rceil \sup \left(D_{1}, D_{1}^{\prime}\right)+\lfloor(D+1) / 2\rfloor \inf \left(D_{1}, D_{1}^{\prime}\right)+D$ en traversant $\lceil(D+1) / 2\rceil$ copies du graphe $G_{1}$ ou $G_{1}^{\prime}$ contenant $x,\lfloor(D+1) / 2\rfloor$ copies du graphe $G_{1}$ ou $G_{1}^{\prime}$ ne contenant pas $x$ et en utilisant $D$ arêtes de la deuxième sorte. Si les sommets $y$ et $y^{\prime}$ sont à la distance $D_{2}$ dans $G_{2}$, le sommet $y$ admet au moins un voisin $y_{1}$, tel que $x=f_{y}\left(y_{1}\right)$ et tel que $y_{1}$ est à distance au plus $D_{2}-1$ de $y^{\prime}$, car $G_{2}$ est biparti. Donc, le sommet $\left(f_{y_{1}}(y), y_{1}\right)$, qui est adjacent à $(x, y)$, est à distance au plus $\left\lceil D_{2} / 2\right\rceil \sup \left(D_{1}, D_{1}^{\prime}\right)+\left\lfloor D_{2} / 2\right\rfloor \inf \left(D_{1}, D_{1}^{\prime}\right)+D_{2}-1$ de $\left(x^{\prime}, y^{\prime}\right)$, ce qui prouve le théorème.

Exemples. La Table 1 ci-dessous donne des exemples d'application quand $G_{2}$ est biparti complet.

Table 1

\begin{tabular}{ccrcccccc}
\hline$G_{1}$ & $G_{1}^{\prime}$ & $n_{1}$ & $n_{1}^{\prime}$ & $n_{2}$ & $n_{2}^{\prime}$ & \multicolumn{1}{c}{$n$} & $\Delta$ & $D$ \\
\hline$K_{3} \times C_{5}$ & $H_{3}^{\prime}$ & 15 & 364 & 392 & 15 & 11340 & 5 & 9 \\
$P \times F_{4}$ & - & 40 & - & - & 40 & 30240 & - & 10 \\
$K_{4}$ & $H_{4} r$ & 4 & 2734 & $n_{1}^{\prime}$ & 12 & 43744 & 6 & 9 \\
$K_{3} \times P_{2} d$ & - & 24 & - & - & 24 & 131232 & - & 10 \\
$K_{5}$ & $H_{5} r$ & 5 & 7817 & - & 15 & 156340 & 7 & 9 \\
$K_{3} \times P_{2} d$ & - & 24 & - & - & 48 & 562824 & - & 10 \\
$K_{6}$ & $H_{7} r$ & 6 & 39223 & - & 24 & 1176690 & 9 & 9 \\
$H S$ & - & 50 & - & - & 100 & 5883450 & - & 10 \\
$K_{6}$ & $H_{5} r$ & 6 & 74906 & - & 30 & 2696616 & 10 & 9 \\
$H S$ & - & 50 & - & - & 150 & 14981200 & - & 10 \\
$K_{7}$ & $H_{4} r$ & 7 & 132869 & - & 35 & 5580498 & 11 & 9 \\
$H S$ & - & 50 & - & - & 200 & 33217250 & - & 10 \\
$P_{4}^{\prime}$ & $H_{11} r$ & 91 & 354323 & - & 283 & 121296802 & 13 & - \\
- & $H_{13} r$ & - & 804401 & - & 465 & 447391446 & 15 & - \\
\hline
\end{tabular}

On peut aussi donner des exemples avec des quadrangles à deux paramètres $Q_{u, v}$, avec $n_{2}=(1+v)(1+u v)$ et $n_{2}^{\prime}=(1+u)(1+u v)$ (voir Payne [17] et Thas [20]). 
Table 2

\begin{tabular}{lllllcrrrr}
\hline$G_{1}$ & $G_{1}^{\prime}$ & $G_{2}$ & $n_{1}$ & $n_{1}^{\prime}$ & $n_{2}$ & $n_{2}^{\prime}$ & $n$ & $\Delta$ & $D$ \\
\hline$K_{1}$ & $P$ & $Q_{3.9}$ & 1 & 10 & 280 & 112 & 1400 & 4 & 8 \\
$K_{1}$ & $H S$ & $Q_{7.49}$ & 1 & 50 & 1720 & 2752 & 154800 & 8 & 8 \\
\hline
\end{tabular}

Table 3

Plus grands $(\Delta, D)$ graphes connus (Octobre 1981)

\begin{tabular}{|c|c|c|c|c|c|c|c|c|c|}
\hline$\Delta D$ & 2 & 3 & 4 & 5 & 6 & 7 & 8 & 9 & 10 \\
\hline & $P$ & $C_{5} \times 4$ & LFQSU & $\mathrm{AL}$ & $\mathrm{H}_{2} \mathrm{r}$ & $\mathrm{H}_{2} i d r$ & Y & Y & $Y$ \\
\hline 3 & & 20 & 34 & 56 & 128 & 158 & 244 & 340 & 536 \\
\hline & $K_{3} \times 5$ & $P \times 4$ & $C_{5} \times 19$ & $H_{3}^{\prime}$ & $\mathrm{H}_{3} r$ & $\mathrm{H}_{3} \mathrm{idr}$ & Thm. 2 & $C_{12} \times C_{161}$ & $C_{\mathrm{s}}(5,9)$ \\
\hline 4 & 15 & 40 & 95 & 364 & 731 & 837 & 1400 & 1932 & 2560 \\
\hline & $K_{3} \times 8$ & $15 \times 4$ & $Q_{4} r$ & $\mathrm{H}_{3}^{\prime} \mathrm{d}$ & $H_{4} r$ & $H_{4} i d r$ & $O_{2.4} d r$ & Thm. 2 & Thm. 2 \\
\hline 5 & & 60 & 174 & 532 & 2134 & 2988 & 5004 & 11340 & 30240 \\
\hline & $K_{1} \times 8$ & $21 \times 5$ & $Q_{j} r$ & $H_{3}^{\prime} d$ & $H_{5} r$ & $H_{4} \times 4$ & $H_{4} \times 6$ & Thm. 2 & Thm. 2 \\
\hline 6 & 32 & 105 & 317 & 756 & 7817 & 10920 & 16380 & 43744 & 131232 \\
\hline & $\mathrm{HS}$ & $24 \times 5$ & $Q_{5} d r$ & $Q_{5} \times 4$ & $\mathrm{H}_{5} d r$ & $H_{5} \times 4$ & $24\left[P_{17}^{\prime}\right]$ & Thm. 2 & Thm. 2 \\
\hline 7 & 50 & & & 1248 & 8998 & $3 \sqrt{248}$ & 54168 & 156340 & 562824 \\
\hline & $P_{7}^{\prime}$ & $\mathrm{HS} \times 4$ & $Q_{7} r$ & $\mathrm{HS}\left[K_{s 1}\right]$ & $H_{7} r$ & $H_{,} i d r$ & Thm. 2 & $H_{5} \times 35$ & $C_{\mathrm{s}}(5,9)$ \\
\hline 8 & 57 & 200 & 807 & 2550 & 39223 & 40593 & 154800 & 273420 & 1310720 \\
\hline & $P_{s}^{\prime} d$ & $Q_{s}^{\prime}$ & $Q_{s} r$ & $\operatorname{HS}\left[K_{101}\right]$ & $H_{s} r$ & $H, \times 4$ & $\operatorname{HS}\left[P_{47}^{\prime} d\right]$ & Thm. 2 & Thm. 2 \\
\hline 9 & 74 & 585 & 1178 & 5050 & 74906 & 156864 & 480250 & $11 / 6690$ & 5883450 \\
\hline 10 & $\begin{array}{l}P_{\prime \prime}^{\prime} \\
91\end{array}$ & $\begin{array}{l}Q_{s}^{\prime} d \\
650\end{array}$ & $\begin{array}{l}Q_{, r} r \\
1629\end{array}$ & $\begin{array}{c}\operatorname{HS}\left[K_{151}\right] \\
7550\end{array}$ & $\begin{array}{c}H_{4}, r \\
132860\end{array}$ & $\begin{array}{c}Q_{: 5}^{\prime}\left[K_{651}\right] \\
380835\end{array}$ & $\begin{array}{l}\mathrm{HS}\left[P_{1190}^{\prime}\right] \\
117550\end{array}$ & $\begin{array}{l}\text { Thm. } 2 \\
2696616\end{array}$ & $\begin{array}{c}\text { Thm. } 2 \\
14981200\end{array}$ \\
\hline & $P_{0}^{\prime}, d$ & $Q_{s}^{\prime} d$ & $Q_{s}^{\prime} \times 5$ & $P_{8}^{\prime}\left[K_{156}\right]$ & $H_{0} d r$ & $Q_{:}^{\prime}\left[K_{1236}\right]$ & $\operatorname{HS}\left[P_{1, \omega]}^{\prime}\right]$ & Thm. 2 & Thm. 2 \\
\hline 11 & 94 & 715 & 2925 & 11388 & 142494 & 723060 & 1990050 & 5580498 & 33217250 \\
\hline & $P_{11}^{\prime}$ & $Q_{s}^{\prime} d$ & $Q_{s}^{\prime} \times 8$ & $P_{0}^{\prime}\left[K_{10,3}\right]$ & $H_{11} r$ & $Q_{s}^{\prime}\left[K_{1521}\right]$ & $P_{s}^{\prime}\left[P_{227}^{\prime}\right]$ & LVLQ & $C_{p}(3,5)$ \\
\hline 12 & 1.33 & 780 & 4680 & 17563 & 354323 & 1065285 & 3778261 & 10077696 & 85887453 \\
\hline & $P_{11}^{\prime} d$ & $Q_{s}^{\prime} d$ & $Q_{s}^{\prime} \times 9$ & $P_{, y}^{\prime}\left[K_{2, s}\right]$ & $H_{11} d r$ & $715\left[K_{2016}\right]$ & $P_{0}^{\prime}\left[P_{2, S 1}^{\prime} d\right]$ & $4680\left[K_{5201}\right]$ & Thm. 2 \\
\hline 13 & 136 & 845 & 5265 & 25844 & 394616 & 1414440 & 7211386 & 24340680 & 121296802 \\
\hline & $P_{13}^{\prime}$ & $Q_{s}^{\prime} d$ & $650 \times 9$ & $P_{11}^{\prime}\left[K_{270}\right]$ & $H_{13} r$ & $910\left[K_{2331}\right]$ & $P_{0,}^{\prime}\left[P_{373}^{\prime}\right]$ & $\left.\frac{4680}{4} K_{9, \mathrm{sis}}\right]$ & $C_{s}(1,10)$ \\
\hline 14 & 183 & 910 & 5850 & 37107 & 804481 & 2130310 & 12694773 & 46243080 & 282475249 \\
\hline 15 & $\begin{array}{l}P_{1,8}^{\prime} d \\
186\end{array}$ & $\begin{array}{l}P_{11}^{\prime} \times 8 \\
1064\end{array}$ & $\begin{array}{c}Q_{s}^{\prime} \times 13 \\
7605\end{array}$ & $\begin{array}{c}P_{111}^{\prime}\left[K_{412}\right] \\
54796\end{array}$ & $\begin{array}{c}H_{13} d r \\
892062\end{array}$ & $\begin{array}{c}H_{13} \times 4 \\
3217872\end{array}$ & $\begin{array}{l}P_{11}^{\prime}\left[P_{403}^{\prime} d\right] \\
22303302\end{array}$ & $\begin{array}{c}4680\left[K_{1.1561}\right] \\
68145480\end{array}$ & $\begin{array}{c}\text { Thm. } 2 \\
447391446\end{array}$ \\
\hline
\end{tabular}




\section{Notations}

\subsection{Graphes particuliers}

$K_{n}: \quad$ graphe complet à $n$ sommets.

$C_{n}: \quad$ cycle à $n$ sommets.

P: $\quad$ graphe de Petersen (10 sommets, $\Delta=3, D=2)$.

HS: $\quad$ graphe d'Hoffman-Singleton (50 sommets, $\Delta=7, D=2$ ).

LFQSU: graphe de Leland, Finkel, Qiao, Solomon et Uhr [15].

AL: $\quad$ graphe de Arden et Lee [1].

$P_{q}$ : $\quad$ graphe d'incidence du plan projectif d'ordre $q$, de degré $q+1$, ayant $2\left(q^{2}+q+1\right)$ sommets et de diamètre 3 : il existe si $q$ est une puissance de premier.

$Q_{q}: \quad$ quadrangle généralisé d'ordre $q$, de degré $q+1$, ayant $2\left(q^{4}-1\right) /(q-1)$ sommets et de diamètre 4 ; il existe si $q$ est une puissance de premier (Benson [2]).

$H_{q}: \quad$ hexagone généralisé d'ordre $q$, de degré $q+1$, ayant $2\left(q^{6}-1\right) /(q-1)$ sommets ế de diamètre 6 ; il existe si $q$ est une puissance de premier (Benson [2]).

$P_{q}^{\prime}: \quad$ graphe quotient de $P_{q}$ par une polarité, graphe de degré $q+1$, ayant $q^{2}+q+1$ sommets et de diamètre 2 .

$Q_{q}^{\prime}: \quad$ graphe quotient de $Q_{q}$ par une polarité, de degré $q+1$, ayant $\left(q^{4}-1\right) /(q-1)$ sommets, de diamètre 3 ; un tel graphe existe s'il existe une polarité, c'est-à-dire pour $q=2^{2 p+1}$ (en particulier, ici, pour $q=8$ : voir Delorme [9]).

$H_{q}^{\prime}$ : $\quad$ graphe quotient de $H_{q}$ par une polarité, de degré $q+1$, ayant $\left(q^{6}-1\right) /(q-1)$ sommets, de diamètre 5 ; un tel graphe existe s'il existe une polarité, c'est-à-dire pour $q=3^{2 p+1}$ (en particulier, ici, pour $q=3$ : voir Delorme [9]).

$O_{p . q}: \quad$ octogone généralisé (Yanushka [21]).

$C_{s}(m, k)$ : graphe de type $C_{s}$ décrit par Delorme et Farhi [10]: ce graphe a un degré maximum $\Delta$, avec $m(\Delta / 2)^{k}$ sommets et de diamètre donné en [10]. Cette famille de graphes est en fait la même que celle introduite par de Bruijn [8] et retrouvée par Golunkov [12] et Lam [13].

LVLQ: famille de graphes décrite par Quisquater [18] et obtenue à partir d'une construction de matrices circulantes généralisées due à Lam et van $\mathbb{L i n t}[14]$. Ces graphes ont un sommet de plus que les graphes $C_{s}(1, D)$.

$C_{p}(m, k)$ : graphe de type $C_{p}$ (associé à un plan projectif) décrit dans Delorme et Farhi [10]. Chaque graphe a un degré maximum $\Delta$, avec $m\left(\Delta^{2} / 4-\Delta / 2+1\right)^{k}$ sommets, où $\Delta / 2-1$ est une puissance de premier. 


\subsection{Opérations de constructions}

$Y: \quad$ construction de $Y$-graphes (voir Quisquater [18]): ces graphes interviennent pour le degré 3 .

$i$ : $\quad$ insertion de sommets sur certaines arêtes (voir $\$ 9$ de Delorme [9]).

$d$ : $\quad$ opération décrite aux $\$ \S 7,8,9$ de Delorme [9]; le cas le plus simple consiste à dédoubler des sommets.

$r$ : opération qui consiste à remplacer un sommet de degré $\Delta$ par un graphe complet $K_{\Delta}$ (voir Quisquater [18]).

$X: \quad$ plusieurs graphes sont obtenus en utilisant un produit spécial défini par Bermond, Delorme et Farhi [4], [5]. Nous les notons $G \times r$, où $G$ désigne le premier graphe et $r$, le nombre de sommets du deuxième. Lorsque $G$ est un graphe de notre table (sans nom spécial), nous le notons par son nombre de sommets.

$G_{1}\left[G_{2}\right]:$ graphe obtenu en appliquant le Théorème 1.

Thm. 2: graphe obtenu en appliquant le Théorème 2.

\section{Note ajouteé à l'épreuve}

Depuis octobre 1981 de nouveaux $(\Delta, D)$ graphes ont été trouvés. Une nouvelle table a été publiée dans Information Processing Letters 15 (1) (1982) 10 -13.

\section{References}

[1] B.W. Arden and H. Lee, A multi-tree-structured network, Proc. Fall COMPCON 78 (1978) 201-210.

[2] C.T. Benson, Minimal regular graphs of girths eight and twelve, Canad. J. Math. 18 (1966) 1091-1094.

[3] J.-C. Bermond and B. Bollobás, Diameters in graphs: a survey, Proc. 12th Southeastern Conf. on Combinatorics, Graph Theory and Computing, Baton Rouge, 1981, to appear.

[4] J.-C. Bermond, C. Delorme and G. Farhi, Large graphs with given degree and diameter II, to appear.

[5] J.-C. Bermond, C. Delorme and G. Farhi, Large graphs with given degree and diameter III, in: B. Bollobás, ed., Proc. Conf. on Graph Theory, Ann. Discrete Math. 13 (1982) pp. $23-32$.

[6] N. Biggs, Algebraic Graph Theory (Cambridge University Press, London, 1974). 1974).

[7] B. Bollobás, Extremal Graph Theory, London Math. Soc. Monograph No. 11 (Academic Press, London, 1978).

[8] N.G. de Bruijn, A combinatorial problem, Koninlijke Nederlandse Academie van Wetenschappen Proc. Ser. A 49 (1946) 758-764.

[9] C. Delorme, Grands graphes de degré et diamètre donnés, à paraître.

[10] C. Delorme and G. Farhi, Large graphs with given diameter I, to appear.

[11] B. Elspas, Topological constraints on interconnection limited logic, Proc. 5th Symp. on Switching Theory and Logical Design, IEEE S-164 (1964) 133-137.

[12] Y.V. Golunkov, Automation program realization of substitutions of symmetric semigroups II, Kibernetika 5 (1975) 35-42. 
[13] C.W.H. Lam, On some solutions of $A^{k}=d I+\lambda J$, J. Comb. Theory, Ser. A 23 (1977) 140-147.

[14] C.W.H. Lam and J.H. van Lint, Directed graphs with unique paths of fixed length, J. Comb. Theory, Ser. B 24 (1978) 331-337.

[15] W. Leland, R. Finkel, L. Qiao, M. Solomon and L. Uhr, High density graphs for processor interconnection, Inform. Process. Lett. 12 (1981) 117-120.

[16] G. Memmi and Y. Raillard, Some new results about the $(d, k)$ graphs problem, IEEE Trans. Comput. to appear.

[17] S.E. Payne, Affine representations of generalized quadrangles, J. Algebra 16 (1970) 473-485.

[18] J.-J. Quisquater, New constructions of large graphs with fixed degree and diameter, to appear.

[19] R.M. Storwick, Improved constructions techniques for $(d, k)$ graphs, IEEE Trans. Comput. 19 (1970) 1214-1216.

[20] J.A. Thas, Combinatorics of finite generalized quadrangles: a survey, Seminar of Geometry and Combinatorics, State University of Ghent, Belgium, Sept. 1980, to appear.

[21] A. Yanushka, On order in generalized polygons, Geometriae Dedicata 20 (1981) 451-458. 